\title{
Indacaterol and glycopyrronium versus indacaterol on body plethysmography measurements in COPD—a randomised controlled study
}

\author{
Joerg Salomon ${ }^{1}$, Daiana Stolz², Guido Domenighetti ${ }^{3}$, Jean-Georges Frey ${ }^{4}$, Alexander J. Turk ${ }^{5}$, Andrea Azzola ${ }^{6}$, \\ Thomas Sigrist ${ }^{7}$, Jean-William Fitting ${ }^{8}$, Ulrich Schmidt ${ }^{9}$, Thomas Geiser ${ }^{10}$, Corinne Wild ${ }^{11}$, Konstantinos Kostikas ${ }^{12}$, \\ Andreas Clemens ${ }^{12^{*}}$ and Martin Brutsche ${ }^{13}$
}

\begin{abstract}
Background: Dual bronchodilator therapy is recommended for symptomatic patients with chronic obstructive pulmonary disease (COPD). There are limited data on effects of a combination of two long-acting bronchodilators on lung function including body plethysmography.

Methods: This multicentre, randomised, double-blind, single-dose, cross-over, placebo-controlled study evaluated efficacy and safety of the free combination of indacaterol maleate (IND) and glycopyrronium bromide (GLY) versus IND alone on spirometric and body plethysmography parameters, including inspiratory capacity (IC), forced expiratory volume in $1 \mathrm{~s}\left(\mathrm{FEV}_{1}\right)$, forced vital capacity (FVC), total lung capacity (TLC) and airway resistance (Raw) in moderate-to-severe COPD patients.
\end{abstract}

Results: Seventy-eight patients with $\mathrm{FEV}_{1} \%$ pred. (mean $\pm \mathrm{SD}$ ) $56 \pm 13 \%$ were randomised. The combination of IND + GLY versus IND presented a numerically higher peak-IC $(\Delta=0.076 \mathrm{~L}, 95 \%$ confidence interval [CI]: $-0.010-0.161 \mathrm{~L}$; $p=0.083)$, with a statistically significant difference in mean IC over $4 \mathrm{~h}(\Delta=0.054 \mathrm{~L}, 95 \% \mathrm{Cl} 0.022-0.086 \mathrm{~L} ; p=0.001)$. $\mathrm{FEV}_{1}, \mathrm{FVC}$ and Raw, but not TLC, were consistently significantly improved by IND + GLY compared to IND alone. Safety profiles of both treatments were comparable.

Conclusion: The free combination of IND + GLY improved lung function parameters as evaluated by spirometry and body plethysmography, with a similar safety profile compared to IND alone.

Trial registration: NCT01699685

Keywords: COPD, Indacaterol, Glycopyrronium, Spirometry, Body plethysmography

\section{Background}

Static lung hyperinflation is one of the significant challenges in patients with COPD. It is characterised by a decrease in the elastic recoil of the lungs with a premature closure of small airways leading to air trapping. The impact on lung function parameters is expressed by an increase in functional residual capacity (FRC) and a progressive decrease in inspiratory reserve volume and

\footnotetext{
* Correspondence: andreas.clemens@novartis.com

${ }^{12}$ Novartis Pharma AG, Basel, Switzerland

Full list of author information is available at the end of the article
}

inspiratory capacity (IC). During exercise, dynamic compression of the airways intensifies and this results in increased dynamic hyperinflation, leading to further exercise limitation [1]. The major clinically relevant mechanism of action of long-acting bronchodilators in COPD is related to the reduction of hyperinflation [1-5], which can be assessed by improvements in IC [6]. Whereas short-acting bronchodilators are used for immediate relief from symptoms, one or more long-acting bronchodilators (long-acting $\beta_{2}$-agonists [LABAs], e.g., indacaterol maleate [IND], and long-acting muscarinic antagonists [LAMAs], e.g., glycopyrronium bromide 
[GLY]) are recommended for long-term maintenance therapy in patients with moderate-to-severe COPD [7]. Since LABAs and LAMAs have different mechanisms of action, they may exert additive bronchodilation effects when used together. This suggests that IND and GLY could be used in combination to optimise and maximise bronchodilation in patients with COPD whose needs are not adequately met by LABA or LAMA monotherapy [8-10]. However, there are limited data on the effects of a combination of two long-acting bronchodilators on body plethysmography lung function parameters in patients with COPD [11].

In this study we evaluated the efficacy and safety of the free combination of IND + GLY versus IND alone on lung function parameters evaluated by body plethysmography, including inspiratory capacity (IC), forced expiratory volume in $1 \mathrm{~s}\left(\mathrm{FEV}_{1}\right)$, forced vital capacity (FVC), total lung capacity (TLC) and airway resistance (Raw), in patients with moderate-to-severe COPD.

\section{Methods}

\section{Study population}

The study was conducted in 11 centres in Switzerland between November 2012 and June 2014, and included a total of 78 eligible patients who were randomised to one of two treatment sequences. The study protocol was reviewed and approved by institutional review boards and ethics committees.

Eligible patients were adults aged $\geq 40$ years with a diagnosis of moderate or severe COPD according to GOLD criteria [12] who had signed an informed consent form, and fulfilling the following: smoking history of at least 10 pack-years [both current and ex-smokers]; post-bronchodilator $\mathrm{FEV}_{1}<80 \%$ and $\geq 30 \%$ of the predicted value, and post-bronchodilator $\mathrm{FEV}_{1} / \mathrm{FVC}<70 \%$. The main exclusion criteria were COPD exacerbations requiring systemic glucocorticoid treatment or antibiotics and/or hospitalisation or a history of respiratory tract infection within 6 weeks prior to screening, concomitant pulmonary disease other than COPD, history of asthma or lung cancer, a known history of alpha-1 antitrypsin deficiency, or a history of hypersensitivity to any of the study medications or to medications from similar drug classes.

\section{Study design and treatment}

This was a multicentre, randomised, double-blind, singledose, cross-over, placebo-controlled study to assess the effect of a single-dose combination of inhaled IND $(150 \mu \mathrm{g})+\mathrm{GLY}(50 \mu \mathrm{g})$ versus inhaled IND $(150 \mu \mathrm{g})+$ placebo (corresponding GLY placebo) on static hyperinflation (Fig. 1). Patients had lung function assessments (spirometry) at each study visit and body plethysmography at Visits 2 and 3. Safety assessments included

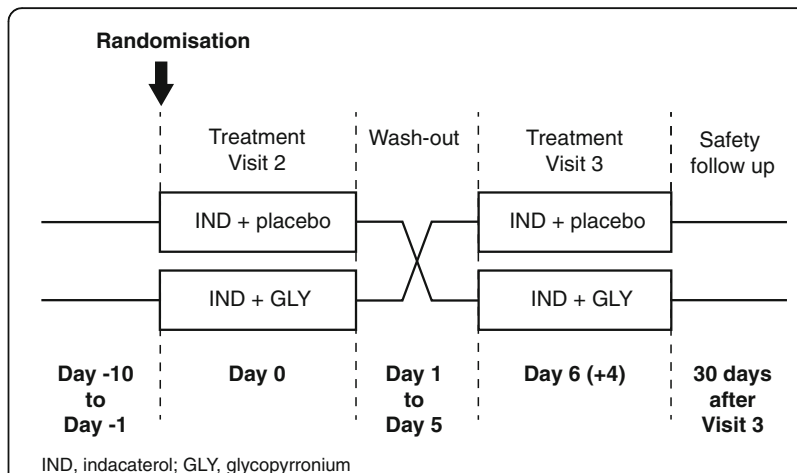

Fig. 1 Study design

physical examinations, vital signs, and monitoring of adverse events (AEs) and serious adverse events (SAEs). All patients prematurely withdrawing from the study underwent study completion evaluations.

\section{Study objectives}

The primary objective was to demonstrate superiority of a single dose of the combined inhalation of IND + GLY versus IND alone on peak-IC, defined as the maximum value within $4 \mathrm{~h}$ of inhalation. The key secondary objective was to compare the efficacy of IND + GLY versus IND in terms of $\mathrm{FEV}_{1}$ over $4 \mathrm{~h}(30,60,120,180$ and $240 \mathrm{~min})$ post dosing. Other secondary objectives were to compare the efficacy of IND + GLY versus IND on IC, FVC, and airway resistance (Raw) over $4 \mathrm{~h}$ (30, 60, 120, 180 and $240 \mathrm{~min}$ ) after dosing.

\section{Statistical analysis}

\section{Sample size calculation}

With regard to peak-IC, a sample size of 69 patients was expected to provide $80 \%$ power to detect a difference of $0.12 \mathrm{~L}$ in $\mathrm{IC}$ at peak between the groups, assuming a standard deviation of differences of $0.35 \mathrm{~L}$ (test level $\alpha=0.025$ one-sided or $\alpha=0.05$ two-sided). Assuming a dropout rate of approximately $10 \%$, a total of $\sim 78$ patients had to be randomised to ensure that at least 70 patients completed the study. Regarding $\mathrm{FEV}_{1}$, a sample size of 70 patients provided $99 \%$ power to detect a difference of $0.18 \mathrm{~L}$ in $\mathrm{FEV}_{1}$ mean values between the groups.

The intention to treat (ITT, full analysis set [FAS]) population consisted of all randomised patients who received at least one dose of study medication and had at least one post-baseline assessment of the primary efficacy variable. The per-protocol (PP) population consisted of all patients in the ITT population without major protocol violations or who discontinued the study due to treatment-related reasons. A supportive analysis on the PP population was performed for the primary endpoint peak-IC and the key secondary endpoint $\mathrm{FEV}_{1}$. The safety population (full 
analysis set; FAS) was defined as all randomised patients who received at least one dose of study medication with at least one post-baseline safety assessment.

Study endpoints were analysed by an analysis of covariance (ANCOVA) model with treatment sequence ( $\mathrm{AB}$ or $\mathrm{BA})$ and treatment as fixed effects, the lung function parameter as a covariate and patient as a random effect. Treatment effect was estimated as the contrast of the treatment effect in the statistical model and presented as point estimates and corresponding 95\% twosided confidence intervals (CIs). The null hypothesis for the primary analysis was that combination of IND + GLY is not superior to IND alone regarding the lung function parameters. The alternative hypothesis was that treatment with a combination of IND + GLY is superior to IND alone. The null hypothesis was rejected in favour of the alternative hypothesis if the $95 \%$ CI of the least squares means treatment contrast of the difference "combination therapy - single therapy" was greater than 0 in its entirety. This corresponds to a planned alpha error of $5 \%$ two-sided or $2.5 \%$ one-sided. An interim analysis was performed after 20 patients had completed Visit 3. No adjustments were needed.

\section{Results}

The mean \pm SD age of the patients was $64.8 \pm 8.4$ years (Table 1), 59.2\% were male, all Caucasian, and 24 (31.2\%) current smokers. Mean time since COPD diagnosis was $5.2 \pm 5.2$ years. The mean $\mathrm{FEV}_{1} \%$ predicted was $56 \pm 13$ and $38.7 \%$ of patients had a GOLD stage of III or above. The mean total lung capacity (TLC) was $120.68 \pm 18.75 \%$ pred. and the mean Raw was $210.99 \pm$ $117.11 \%$ pred. The patient disposition and randomisation is given in Fig. 2.

The combination of IND + GLY versus IND presented a numerically higher peak-IC (2.95 L versus $2.88 \mathrm{~L})$, with an adjusted treatment difference $(\Delta)$ of $0.076 \mathrm{~L}(95 \%$ $-0.010-0.161 \mathrm{~L} ; p=0.083$ ) (Fig. 3a). IND + GLY presented also a statistically significant difference in mean IC over $4 \mathrm{~h}$ versus IND $(2.76 \mathrm{~L}$ versus $2.70 \mathrm{~L} ; \Delta=$ $0.054 \mathrm{~L}, 95 \%$ CI $0.022-0.086 \mathrm{~L} ; p=0.001$ ) (Fig. 3b). $\mathrm{FEV}_{1}$, FVC and Raw, but not TLC, were significantly improved by IND + GLY compared to IND alone. A statistically significant adjusted treatment difference in $\mathrm{FEV}_{1}$ was noted at all time points in favour of IND + GLY treatment ( $p<0.001$ for all comparisons), reaching a peak difference of $\Delta=0.099 \mathrm{~L}(95 \% \mathrm{CI} 0.060-0.139 \mathrm{~L})$ at 120 min post-dose (Fig. 4a). Similarly, IND + GLY resulted in higher FVC mean values at all time points after a single-dose inhalation ( $p<0.01$ for all comparisons), reaching a peak difference of $\Delta=0.163 \mathrm{~L}$ (95\%CI 0.092 $0.234 \mathrm{~L}$ ) at 240 min post-dose (Fig. 4b). Raw measurements were consistently lowered by IND + GLY treatment at all time points after the single-dose inhalation
Table 1 Demography and baseline characteristics (ITT population, $N=76)$

\begin{tabular}{|c|c|c|}
\hline & & Mean (SD), $N=76$ \\
\hline \multicolumn{2}{|l|}{ Age at informed consent, years } & $64.80(8.39)$ \\
\hline \multicolumn{2}{|l|}{ Height, cm } & $168.22(8.55)$ \\
\hline \multicolumn{2}{|l|}{ Weight, kg } & $75.45(15.52)$ \\
\hline \multicolumn{2}{|l|}{$\mathrm{BMI}, \mathrm{kg} / \mathrm{m}^{2}$} & $26.65(5.05)$ \\
\hline \multicolumn{2}{|l|}{$\begin{array}{l}\text { Smoking history } \\
\text { Number of pack-years, years }\end{array}$} & $50.13(23.28)$ \\
\hline \multicolumn{2}{|l|}{ Years since COPD diagnosis } & $5.17(5.24)$ \\
\hline \multicolumn{2}{|l|}{ Age at COPD diagnosis, years } & $60.16(10.96)$ \\
\hline \multicolumn{2}{|l|}{$\mathrm{FEV}_{1} \%$ predicted } & $56.09(13.28)$ \\
\hline \multicolumn{2}{|l|}{$\mathrm{FEV}_{1}{ }^{*}, \mathrm{~L}$} & $1.50(0.45)$ \\
\hline \multicolumn{2}{|l|}{$F V C^{*}, L$} & $2.95(0.89)$ \\
\hline \multicolumn{2}{|l|}{$I C, L$} & $2.45(0.69)$ \\
\hline \multicolumn{2}{|l|}{ Total Lung Capacity (TLC), L } & $7.13(1.42)$ \\
\hline \multicolumn{2}{|l|}{ TLC \% of predicted normal value } & $120.68(18.75)$ \\
\hline \multicolumn{2}{|l|}{ Airway Resistance (Raw) } & $6.73(3.19)$ \\
\hline \multicolumn{2}{|l|}{ Raw $\%$ of predicted normal value } & $210.99(117.11)$ \\
\hline \multirow{2}{*}{\multicolumn{2}{|c|}{ Hyperinflation IC/TLC }} & $0.35(0.09)$ \\
\hline & & N (\%) \\
\hline \multirow[t]{2}{*}{ Gender } & Male & $45(59.2)$ \\
\hline & Female & $31(40.8)$ \\
\hline \multirow{3}{*}{$\begin{array}{l}\text { Number of patients with } \\
\text { current medical condition }\end{array}$} & CAD & $7(9.2)$ \\
\hline & Hypertension & $29(38.2)$ \\
\hline & Diabetes mellitus & $4(5.3)$ \\
\hline \multirow{4}{*}{$\begin{array}{l}\text { Number of patients } \\
\text { according COPD GOLD-stage }\end{array}$} & Stage I & $8(10.5)$ \\
\hline & Stage ॥ & $31(40.8)$ \\
\hline & Stage III & $30(39.5)$ \\
\hline & Stage IV & $7(9.2)$ \\
\hline
\end{tabular}

$I T$ intention to treat, $N / n$ number of patients, $B M I$ body mass index, $S D$ standard deviation, COPD chronic obstructive pulmonary disease, $F E V_{1}$ forced expiratory volume in $1 \mathrm{~s}, F V C$ forced vital capacity, IC inspiratory capacity, CAD coronary artery disease, GOLD stage defined as: stage I $=\mathrm{FEV} / \mathrm{FVC}<70 \%$ and $\mathrm{FEV}_{1} \geq 80 \%$ predicted; stage $\mathrm{II}=\mathrm{FEV}_{1} / \mathrm{FVC}<70 \%$ and $50 \% \leq \mathrm{FEV}_{1}<80 \%$ predicted; stage III $=\mathrm{FEV}_{1} / \mathrm{FVC}<70 \%$ and $30 \% \leq \mathrm{FEV} 1<50 \%$ predicted; stage $\mathrm{IV}=\mathrm{FEV}_{1} / \mathrm{FVC}<70 \%$ and $\mathrm{FEV}_{1}<30 \%$ predicted ${ }^{*} N=75$

( $\mathrm{p}<0.001$ for all comparisons), reaching a peak difference of $\Delta=-0.667 \mathrm{cmH}_{2} \mathrm{O} / \mathrm{L} / \mathrm{sec} \quad(95 \% \mathrm{CI}-0.928--0.406$ $\mathrm{cmH} 2 \mathrm{O} / \mathrm{L} / \mathrm{sec}$ ) at $240 \mathrm{~min}$ post-dose (Fig. 4c), in favour of dual bronchodilation $(p \leq 0.001)$. There were no differences in TLC between the study treatments.

\section{Safety}

Eight (10.4\%) patients experienced treatment-emergent adverse events (TEAEs) (Table 2). No patient died in the course of the study or experienced any treatment-emergent SAE. According to the investigators' assessment, a relation to study medication was not suspected for any of the TEAEs. The intensity of TEAEs was mostly mild 


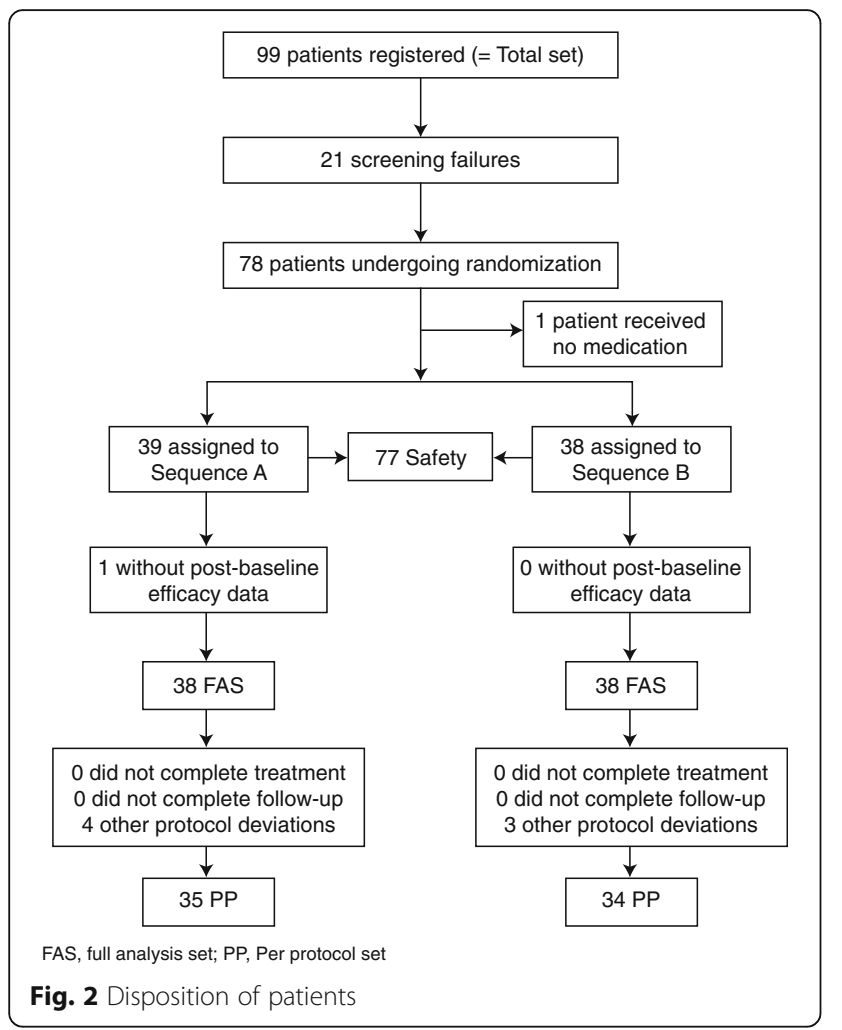

(6 patients) or moderate ( 2 patients). Prior to the first dose of study medication, one patient experienced atrial fibrillation of moderate intensity. In conclusion, the treatments were well tolerated with a good safety profile.

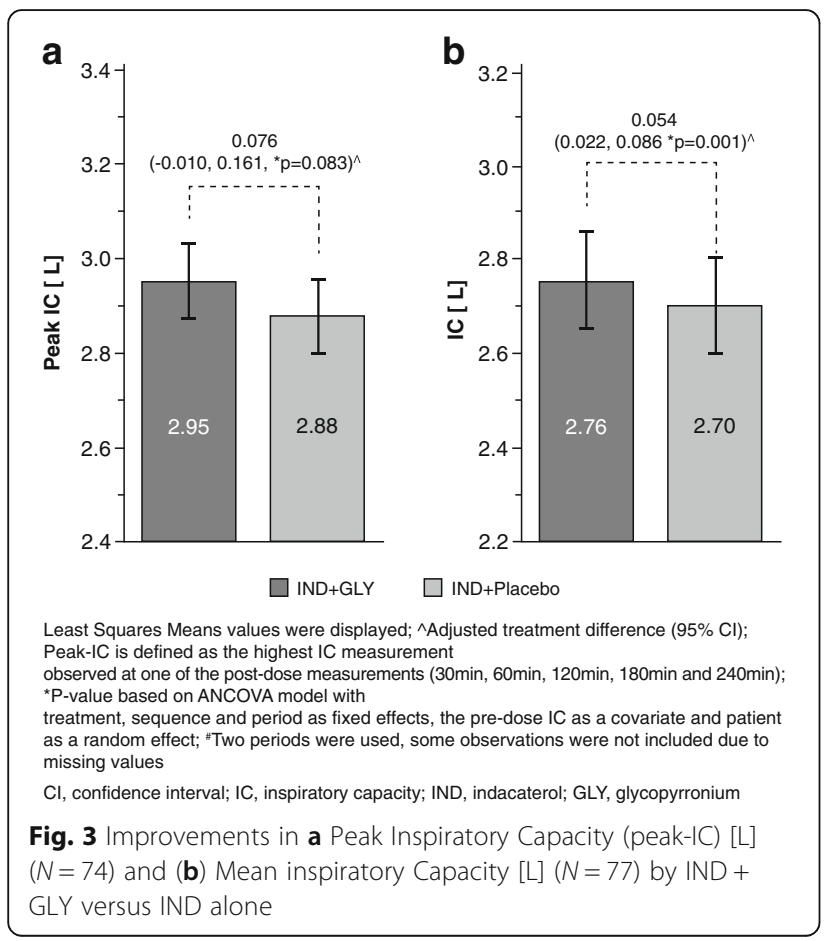

\section{Discussion}

In this prospective, randomised study we showed that the combination of two long-acting bronchodilators provided a greater improvement in lung hyperinflation and lung function parameters compared to a single long-acting agent. Specifically, IND + GLY provided a numerical improvement in peak-IC combined with a statistically significant difference in mean IC over $4 \mathrm{~h}$ compared to IND monotherapy. Additionally, the treatment with IND + GLY resulted in consistent statistically significant improvements in $\mathrm{FEV}_{1}, \mathrm{FVC}$ and Raw compared to IND alone. The two treatments presented a similar safety profile.

As a unique feature of the trial, the use of body plethysmography allowed us to observe the significant difference in Raw in favour for IND + GLY in this study. Raw is not frequently reported in studies evaluating the effect of bronchodilators in COPD. However, this parameter is suggested to be sensitive and to reflect airflow obstruction, particularly of the peripheral airways, more accurately than the $\mathrm{FEV}_{1} / \mathrm{FVC}$ ratio. In assessing the acute functional effect of bronchodilators, specific Raw change-based criteria may be preferable to $\mathrm{FEV}_{1^{-}}$or FVC-based criteria, being more closely related to bronchodilator-induced improvements in lung mechanics and dyspnoea at rest [13]. Raw measurements were strongly improved by IND + GLY treatment compared to IND monotherapy at all time points after single-dose inhalation.

A possible explanation of the non-statistically significant result in SYNERGY on peak-IC might be attributed to the high variability of this measurement. This is supported by the fact that in contrast to the peak-IC measurement, the adjusted mean IC in the SYNERGY study (which included several values) presented a statistically significant difference between the two treatments. Additionally, the results of the present study are consistent with those of other published studies that have investigated the efficacy and safety of LABA/LAMA combination therapy in patients with COPD [7, 11, 14-18]. In order to allow for higher power and better generalisability of the results, we additionally evaluated with a similar analysis as in SYNERGY the peak-IC and $\mathrm{FEV}_{1}$ in a pooled analysis of patient-level data $(n=1,548)$ from 3 studies that evaluated the combination of IND + GLY versus IND, i.e. SYNERGY (present study), SHINE [14] and GLOW6 [7] (see details in the Additional file 1 Online Supplement). Mean adjusted peak-IC in this pooled analysis was statistically significantly higher for patients treated with IND + GLY versus IND alone $(\Delta=0.075 \mathrm{~L} ; 95 \%$ CI $0.040-0.109 \mathrm{~L} ; p \leq 0.001)$ (Additional file 2 Figure S1). Additionally, $\mathrm{FEV}_{1}$ was statistically significantly higher for IND + GLY versus IND at 30,120 and $240 \mathrm{~min}$ after a single dose inhalation, with a maximal difference at $120 \mathrm{~min}(\Delta=0.094 \mathrm{~L}$; $95 \%$ 


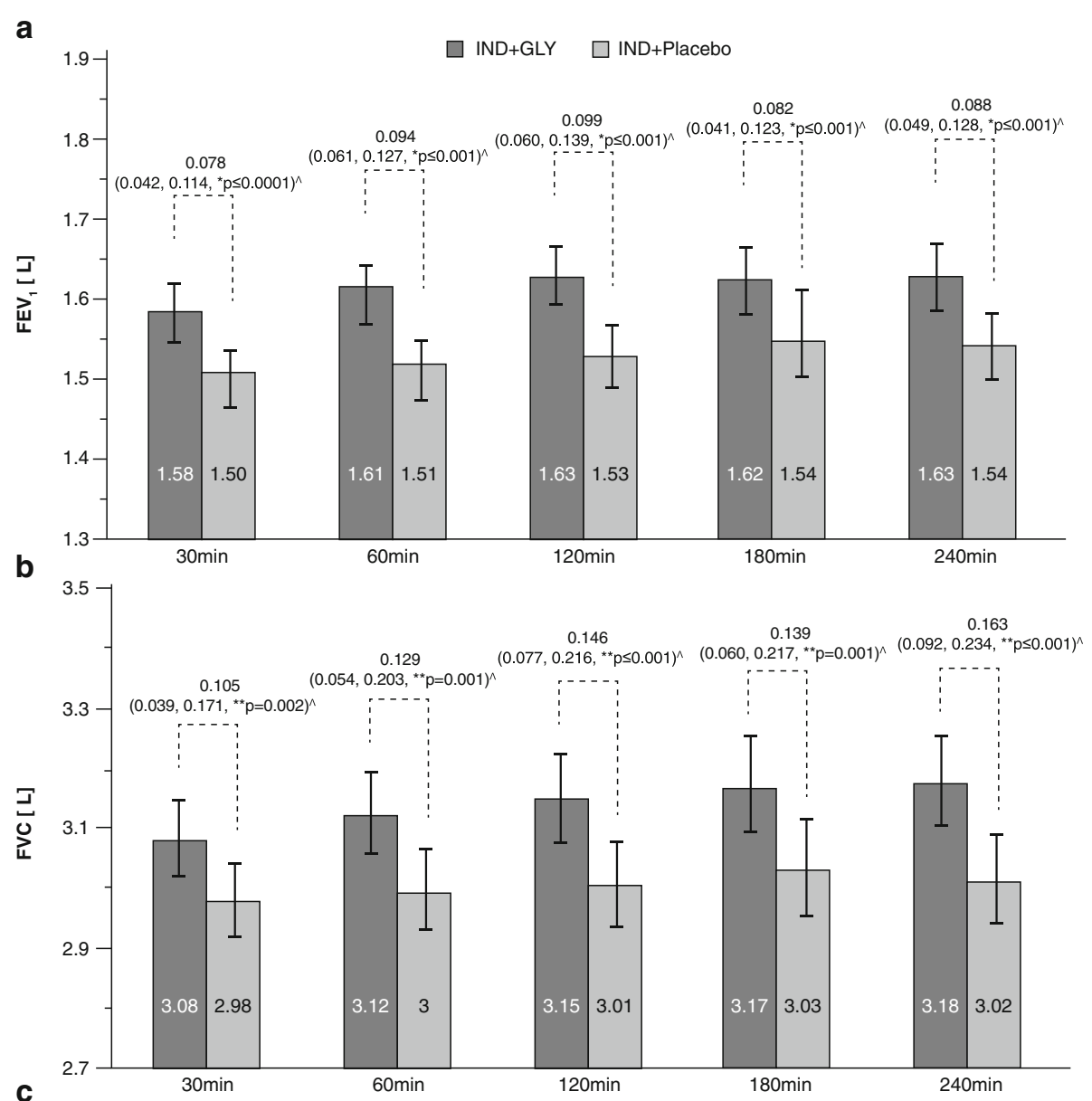

C

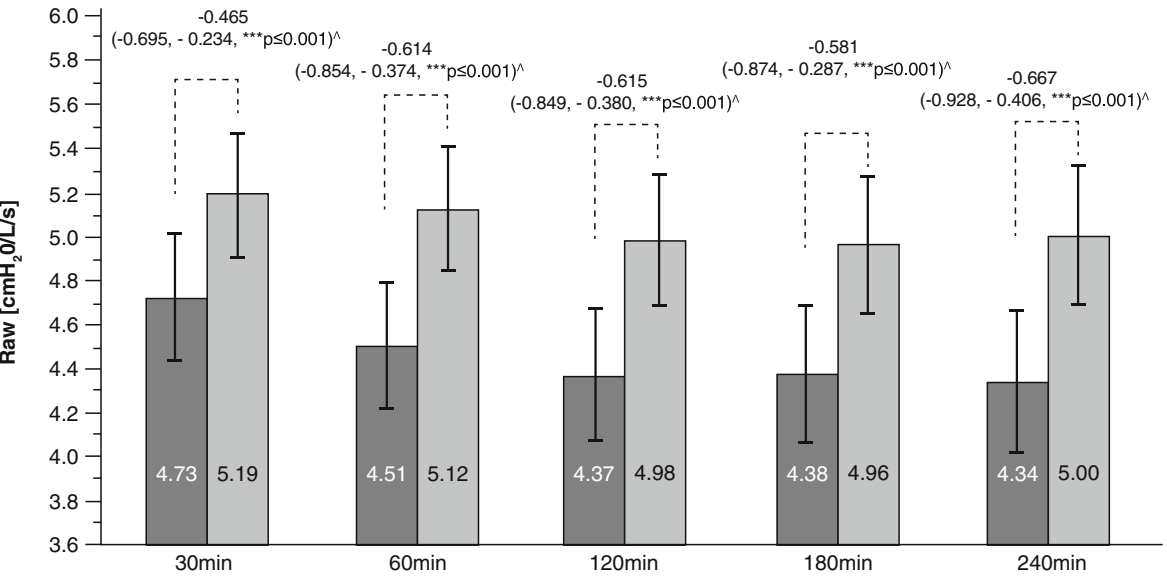

Least Squares Means values were displayed; ^Adjusted treatment difference $(95 \% \mathrm{Cl}) ;{ }^{*} \mathrm{P}$-value based on ANCOVA model with treatment, sequence and period as fixed effects, the pre-dose $\mathrm{FEV}_{1}$ as a covariate and patient as a random effect; ${ }^{* *} \mathrm{P}$-value based on ANCOVA model with treatment, sequence and period as fixed effects, the pre-dose FVC as a covariate and patient as a random effect; ${ }^{* * *} \mathrm{P}$-value based on ANCOVA model with treatment, sequence and period as fixed effects, the pre-dose Raw as a covariate and patient as a random effect

$\mathrm{Cl}$, confidence interval; IND, indacaterol; GLY, glycopyrronium

Fig. 4 a Forced expiratory volume in $1 \mathrm{~s}$ (FEV1) [L] over time (ITT population, $N=77)$; b Forced vital capacity (FVC) [L] (N= 77); c Airway resistance (Raw) $[\mathrm{cmH} 2 \mathrm{O} / \mathrm{L} / \mathrm{s}](\mathrm{N}=77)$

CI $0.076-0.112 \mathrm{~L} ; p \leq 0.001$ ) (Additional file 3 Figure S2). These results further support the reduction of static hyperinflation, as expressed by IC, by a combination of two bronchodilators compared to a single agent.
The physiological and clinical significance of these results can be attributed to prolonged maximal bronchodilation that minimises air trapping and leads to effective reduction of static and dynamic lung 
Table 2 Incidence of TEAEs by primary system organ class (safety population, $N=77$ )

\begin{tabular}{|c|c|c|c|}
\hline & Combined treatment $(I N D+G L Y)$ & IND alone (IND + placebo) & All \\
\hline Primary system organ class & $N=77, \mathrm{n}(\%)$ & $N=77, \mathrm{n}(\%)$ & $N=77, \mathrm{n}(\%)$ \\
\hline Number (\%) of patients with at least one AE & $5(6.5)$ & $3(3.9)$ & $8(10.4)$ \\
\hline Investigations & $1(1.3)$ & $2(2.6)$ & $3(3.9)$ \\
\hline Respiratory, thoracic \& mediastinal disorders & $2(1.3)$ & $1(1.3)$ & $3(3.9)$ \\
\hline Infections \& infestations & $1(1.3)$ & 0 & $1(1.3)$ \\
\hline Musculoskeletal and connective tissue disorders & $1(1.3)$ & 0 & $1(1.3)$ \\
\hline
\end{tabular}

TEAEs treatment-emergent adverse events, IND indacaterol, IND +GLY indacaterol and glycopyrronium, $N$ or $n$ number of patients, $A E$ adverse event

hyperinflation. Improved IC is associated with improved exercise endurance and dyspnoea [2,3] and potentially improved long-term outcomes. Casanova et al. showed that lung hyperinflation, as expressed by the IC/TLC ratio, is an independent predictor of mortality [19]. Furthermore, Tantucci et al. identified IC as a powerful functional predictor of all-cause and respiratory mortality and of exacerbation-related hospital admissions in patients with COPD [20].

The improvement in bronchodilation and measures of hyperinflation observed in the present study is supported by data from the BRIGHT study (IND/GLY fixed-dose combination versus placebo and tiotropium), which showed significantly improved dynamic IC, trough $\mathrm{FEV}_{1}$, residual volume (RV) and FRC in patients with moderate-to-severe COPD receiving IND/GLY that were accompanied by increased exercise endurance [11]. Mahler et al. showed that IND + tiotropium provided greater bronchodilation and lung deflation compared with tiotropium monotherapy [17]. To what extent these effects have a clinically significant impact on outcomes other than lung function and exercise endurance requires further evaluation. However, there is significant evidence that exacerbations, the relevant trigger for progression, are more effectively prevented by IND + GLY than by a single long-acting bronchodilator [21].

We acknowledge that there were limitations in the study. These include the cross-over study design, the short study duration, and the potentially limited patient population due to the clinical trial settings. Additionally, we need to acknowledge that in patients with severe airflow limitation, the plethysmographic Raw may be of limited validity. Finally, post hoc it became obvious that possibly the initially taken assumptions for the power calculations were overestimated, resulting in a relatively small sample size to reach statistical significance. This is supported by the results of the pooled analysis showing the statistical significance for peak-IC.

In our study all treatments were equally well tolerated and showed a good safety profile, which is also documented in multiple clinical trials and the use in clinical practice $[7,8,10,11,14,16,17,21]$.

\section{Conclusions}

In summary, the results of the present study show that treatment with IND + GLY had a stronger beneficial effect on lung hyperinflation and airflow obstruction parameters in patients with COPD than treatment with IND alone. The treatment was well tolerated and had a good safety profile. These data support the use of dual bronchodilator therapy to not only improve airway calibre $\left(\mathrm{FEV}_{1}\right)$ but also decrease hyperinflation and its associated negative consequences in patients with COPD.

\section{Additional files}

Additional file 1: Online supplement. (DOCX $182 \mathrm{~kb})$

Additional file 2: Figure S1. Peak Inspiratory Capacity [L] - pooled analysis of SYNERGY, SHINE and GLOW6 $(N=1538)^{\#}$. (PDF $\left.376 \mathrm{~kb}\right)$

Additional file 3: Figure S2. Forced expiratory volume in $1 \mathrm{~s}$ (FEV1) [L] pooled analysis of SYNERGY, SHINE and GLOW6 (N=1503). (PDF 371 kb)

\section{Abbreviations}

AEs: Adverse events; ANCOVA: Analysis of covariance; Cl: Confidence interval; COPD: Chronic obstructive pulmonary disease; FAS: Full analysis set; $\mathrm{FEV}_{1}$ : Forced expiratory volume in $1 \mathrm{~s}$; FRC: Functional residual capacity; FVC: Forced vital capacity; GLY: Glycopyrronium bromide; IC: Including inspiratory capacity; IND: Indacaterol maleate; IND: Indacaterol maleate; ITT: Intention to treat; LABAs: Long-acting $\beta 2$-agonists; LAMAs: Long-acting muscarinic antagonists; PP: Per-protocol; SAEs: Serious adverse events; TEAEs: Treatment-emergent adverse events; TLC: Total lung capacity

\section{Acknowledgements}

The study was sponsored by Novartis Pharma Schweiz AG. The authors thank the physician investigators who contributed to patient enrolment, together with the nursing and technical staff at each participating centre. For statistical support in the pooled analysis, the authors thank Giovanni Bader from Novartis Pharma AG, Switzerland. The clinical trial was conducted in collaboration with THERAMetrics (previously Pierrel Research), who monitored the conduct of the study, performed randomisation and were responsible for the collection of the data. The authors were assisted in the preparation of the manuscript by THERAMetrics (previously Pierrel Research) and Rohit Bhandari (professional medical writer; Novartis) for assistance in the preparation of this manuscript. Writing support was funded by Novartis Pharma AG, Switzerland.

\section{Funding}

Study was funded by Novartis Pharma AG, Switzerland.

Writing support was funded by Novartis Pharma AG, Switzerland. 


\section{Availability of data and materials}

Trial was registered at clinicaltrials.gov (NCT01699685). The datasets used and/or analysed during the current study available from the corresponding author on reasonable request. All data generated or analysed during this study are included in this published article and its supplementary information files.

\section{Authors' contributions}

All authors have provided substantial contribution for the study conception and design, acquisition of data, or analysis and interpretation of data. All authors were involved in drafting/revising this manuscript for important intellectual content and have given final approval of the version to be published.

\section{Competing interests}

Corinne Wild (CW) is a full-time employee of Novartis Pharma Schweiz AG. Andreas Clemens (AC) and Konstantinos Kostikas (KK) are full-time employees and shareholders of Novartis Pharma AG. Thomas Geiser (TG) has received advisory board and speaker fees from Novartis. Joerg Salomon (JS), JeanWilliam Fitting (J-WF), Thomas Sigrist (TS), Jean-Georges Frey (J-GF), Guido Domenighetti (GD) and Daiana Stolz (DS), Alexander J. Turk (AT), Andrea Azzola (AA), Ulrich Schmidt (US) and Martin Brutsche (MB) have no conflict of interests related to this manuscript.

\section{Consent for publication}

\section{Not applicable.}

\section{Ethics approval and consent to participate}

The study protocol and all amendments were reviewed by the Independent Ethics Committee or Institutional Review Board for each center. Lead Ethic Committee: Ethikkommission des Kantons St. Gallen (Reference Number: EKSG 12/093/L/1B). Sub-ethic committees: Kantonale Ethikkommission Bern (Reference Number: 161/12), Comitato etico cantonale (Ticino) (Reference Number: Rif. CE 2618), Commission cantonale valaisanne d'éthique médicale (Reference Number: CCVEM 037/12), Commission cantonale d'éthique de la recherche sur l'être humain (Reference Number: 334/12), Kantonale Ethikkommission (Aargau) (Reference Number: 2012/062), Kantonale Ethikkommission Basel (Reference Number: 246/12), and Kantonale Ethikkommission Zürich (Reference Number: KEK-ZH-Nr. 2012-0396). This trial is registered at ClinicalTrials.gov (NCT01699685). The study was conducted in accordance with the ethical principles of the Declaration of Helsinki. Written informed consent was obtained from all patients.

\section{Summary}

Indacaterol and glycopyrronium showed a stronger beneficial effect on body plethysmography measurements in patients with COPD than indacaterol alone.

\section{Author details}

${ }^{1}$ Lung Centre Salem-Spital, Bern, Switzerland. ${ }^{2}$ University Hospital Basel, Basel, Switzerland. ${ }^{3}$ Regional Hospital La Carità, Locarno, Switzerland. ${ }^{4}$ Hospital du Valais, Sion, Switzerland. ${ }^{5}$ Hospital, Zürcher Rehazentrum Wald, Wald, Switzerland. ${ }^{6}$ Regional Hospital Civico, Lugano, Switzerland. ${ }^{7}$ Hospital, Klinik Barmelweid, Barmelweid, Switzerland. ${ }^{8}$ Lausanne University Hospital, Lausanne, Switzerland. ${ }^{9}$ Kliniken Valens, Rehabilitation Centre, Walenstadtberg, St. Gallen, Switzerland. ${ }^{10}$ University Hospital of Bern, Bern, Switzerland. ${ }^{11}$ Novartis Pharma Schweiz AG, Rotkreuz, Switzerland. ${ }^{12}$ Novartis Pharma AG, Basel, Switzerland. ${ }^{13}$ Cantonal Hospital, St. Gallen, Switzerland.

\section{Received: 16 November 2016 Accepted: 5 December 2016}

Published online: 11 January 2017

\section{References}

1. Rossi A, Aisanov Z, Avdeev S, Di Maria G, Donner CF, Izquierdo JL, et al. Mechanisms, assessment and therapeutic implications of lung hyperinflation in COPD. Respir Med. 2015;109:785-802.

2. O'Donnell DE. Hyperinflation, dyspnea, and exercise intolerance in chronic obstructive pulmonary disease. Proc Am Thorac Soc. 2006;3:180-4.

3. O'Donnell DE, Laveneziana P. The clinical importance of dynamic lung hyperinflation in COPD. COPD. 2006;3:219-32.
4. Macklem PT. Therapeutic implications of the pathophysiology of COPD. Eur Respir J. 2010:35:676-80.

5. Kostikas K, Siafakas NM. Does the Term "Deflators" Reflect More Accurately the Beneficial Effects of Long-acting Bronchodilators in COPD? COPD. 2016; 13:537-9.

6. Rossi A, Centanni S, Cerveri I, Gulotta C, Foresi A, Cazzola M, et al. Acute effects of indacaterol on lung hyperinflation in moderate COPD: a comparison with tiotropium. Respir Med. 2012;106:84-90.

7. Vincken W, Aumann J, Chen H, Henley M, McBryan D, Goyal P. Efficacy and safety of coadministration of once-daily indacaterol and glycopyrronium versus indacaterol alone in COPD patients: the GLOW6 study. Int J Chron Obstruct Pulmon Dis. 2014;9:215-28.

8. Bateman ED, Mahler DA, Vogelmeier CF, Wedzicha JA, Patalano F, Banerji D. Recent advances in COPD disease management with fixed-dose long-acting combination therapies. Expert Rev Respir Med. 2014:8:357-79.

9. Calzetta L, Matera MG, Cazzola M. Pharmacological interaction between LABAs and LAMAs in the airways: optimizing synergy. Eur J Pharmacol. 2015;761:168-73.

10. Pelaia G, Maselli R, Matera MG. Treatment of chronic obstructive pulmonary disease by dual bronchodilation with coformulation of indacaterol/ glycopyrronium. Pharmacology. 2014;94:249-58.

11. Beeh KM, Korn S, Beier J, Jadayel D, Henley M, D'Andrea P, et al. Effect of QVA149 on lung volumes and exercise tolerance in COPD patients: the BRIGHT study. Respir Med. 2014;108:584-92.

12. GOLD. Global Initiative for Chronic Obstructive Lung Disease (GOLD). Global Strategy for the Diagnosis, Management and Prevention of COPD, Global Initiative for Chronic Obstructive Lung Disease GOLD 2010. Available from www.goldcopd.com . Accessed 23 June, 2016.

13. Santus P, Radovanovic D, Henchi S, Di Marco F, Centanni S, D'Angelo E, et al. Assessment of acute bronchodilator effects from specific airway resistance changes in stable COPD patients. Respir Physiol Neurobiol. 2014; 197:36-45.

14. Bateman ED, Ferguson GT, Barnes N, Gallagher N, Green Y, Henley M, et al. Dual bronchodilation with QVA149 versus single bronchodilator therapy: the SHINE study. Eur Respir J. 2013:42:1484-94.

15. Ferguson GT, Flezar M, Korn S, Korducki L, Gronke L, Abrahams R, et al. Efficacy of tiotropium + olodaterol in patients with chronic obstructive pulmonary disease by initial disease severity and treatment intensity: a post Hoc analysis. Adv Ther. 2015:32:523-36.

16. Mahler DA, Decramer M, D'Urzo A, Worth H, White T, Alagappan VK, et al. Dual bronchodilation with QVA149 reduces patient-reported dyspnoea in COPD: the BLAZE study. Eur Respir J. 2014;43:1599-609.

17. Mahler DA, D'Urzo A, Bateman ED, Ozkan SA, White T, Peckitt C, et al Concurrent use of indacaterol plus tiotropium in patients with COPD provides superior bronchodilation compared with tiotropium alone: a randomised, double-blind comparison. Thorax. 2012;67:781-8.

18. Yoshiki I, Kazuyuki C, Akihiro T, Yumeko H, Ryo A, Tomoe F, et al. Add-On Indacaterol Further Improved Lung Function, Airway Resistance, Exercise-Endurance And CAT Score In The COPD Patients Already Treated With Tiotropium. C50 CHRONIC OBSTRUCTIVE PULMONARY DISEASE PHARAMCOTHERAPY: OXYGEN, MECHANISMS, AND REAL LIFE STUDIES. American Thoracic Society International Conference Abstracts: American Thoracic Society; 2013. p. A4368-A.

19. Casanova C, Cote C, de Torres JP, Aguirre-Jaime A, Marin JM, Pinto-Plata V, et al. Inspiratory-to-total lung capacity ratio predicts mortality in patients with chronic obstructive pulmonary disease. Am J Respir Crit Care Med. 2005;171:591-7.

20. Tantucci C, Donati P, Nicosia F, Bertella E, Redolfi S, De Vecchi M, et al. Inspiratory capacity predicts mortality in patients with chronic obstructive pulmonary disease. Respir Med. 2008;102:613-9.

21. Wedzicha JA, Decramer M, Ficker JH, Niewoehner DE, Sandstrom T, Taylor AF, et al. Analysis of chronic obstructive pulmonary disease exacerbations with the dual bronchodilator QVA149 compared with glycopyrronium and tiotropium (SPARK): a randomised, double-blind, parallel-group study. Lancet Respir Med. 2013;1:199-209. 